\title{
Construction of an Immune-related Gene Model for Predicting Prognosis and Immune Infiltration in Clear Cell Renal Cell Carcinoma
}

\section{Fengping Ji}

First Affiliated Hospital of Zhengzhou University

Xin Liu

First Affiliated Hospital of Zhengzhou University

\section{Yanping Zhang}

First Affiliated Hospital of Zhengzhou University

Erpeng Liu

First Affiliated Hospital of Zhengzhou University

Jianguo Wen ( $\nabla$ wenjianguo2020@126.com )

First Affiliated Hospital of Zhengzhou University

\section{Research Article}

Keywords: Clear cell renal cell carcinoma, immune-related gene, prognostic model, immune microenvironment, immunotherapy

Posted Date: January 5th, 2022

DOI: https://doi.org/10.21203/rs.3.rs-1186623/v1

License: (c) (1) This work is licensed under a Creative Commons Attribution 4.0 International License. Read Full License 


\section{Abstract}

Background: Clear cell renal cell carcinoma (ccRCC) is a common pathological type of kidney cancer with high immune infiltration that has been proven to be treatable with immune checkpoint inhibitor (ICI) therapy. However, the role of immunity in ccRCC remains poorly understood. Therefore, this paper aimed

to develop and validate a novel immune-related prognostic marker to predict both the overall survival rate (OS) of cCRCC patients and the response to ICI therapy.

Methods: Based on the transcriptome and clinicopathological data of ccRCC from The Cancer Genome Atlas (TCGA) dataset and immune-related genes (IRGs) from immune datasets, IRGs related to prognosis were screened to construct an IRG prognostic index (IRGPI) via coexpression analysis and Cox regression. After verifying that IRGPI was a prognostic indicator independent of clinical parameters, a nomogram was established. In addition, functional enrichment analysis, the CIBERSORT algorithm and singlesample gene set enrichment analysis (sSGSEA) were performed to compare the molecular and immune characteristics of IRGPI-defined subgroups. Finally, the expression of immunosuppressive genes, tumor mutational burden (TMB) and the TIDE algorithm were used to predict the response of ICI therapy in different IRGPI subgroups.

Results: A total of 11 IRGs (IFNG, XCL1, APOBEC3G, CD86, CXCR3, IL10RA, IL2RG, CD244, SH2D1A, CD3D

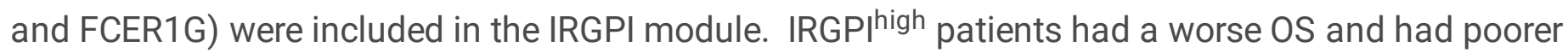
clinical pathological status than IRGPI ${ }^{\text {low }}$ patients. A nomogram containing clinical features and IRGPI scores may guide the clinical practice of ccRCC. Chemokine signaling pathways were mainly involved in functional enrichment analysis. Furthermore, the IRGPI could effectively reflect the immune characteristics and immune checkpoint gene expression of cCRCC and the response to ICl therapy.

Conclusions: The IRGPI is a promising biomarker for determining prognosis and has the potential to be used to predict immunotherapy response in ccRCC.

\section{Introduction}

Clear cell renal cell carcinoma (ccRCC) is a common pathological type of kidney cancer, representing almost $70 \%-80 \%$ of pathological cases. Approximately $20-30 \%$ of ccRCC progresses to metastasis quickly ${ }^{1}$, which makes treatment challenging. $\mathrm{ccRCC}$ is a disease without a cure, and the effectiveness of surgery, radiotherapy, and chemotherapy is limited at the metastatic or advanced stage. Compared to conventional therapies, novel targeted agents, immune checkpoint inhibitor $(\mathrm{ICI})$ treatments and combination therapy strategies have revolutionized the management of $\operatorname{ccRCC}^{2,3}$. However, only a subset of patients achieved durable responses when treated with ICls, which may be attributed to changes in dissimilar biochemical parameters and a high level of cancer heterogeneity caused by changes in the tumor immune microenvironment (TIME). 
TIMEs, such as immune cell compositions and tumor-infiltrating lymphocyte (TIL) status, also affect tumor immunity. Although growing evidence has highlighted the TIME as an important prognostic indicator that can enhance the potential of precision immunotherapy, its clinical implication on ccRCC patient survival is not fully understood ${ }^{4-6}$. Promising biomarkers for determining prognosis and predicting immunotherapy response in ccRCC are urgently needed ${ }^{7}$. With the establishment of The Cancer Genome Atlas (TCGA) database and the development of gene sequencing technology, it has become possible to comprehensively analyze the immunogenomic landscape of ccRCC.

In this study, immune-related genes (IRGs) related to prognosis were identified via weighted gene coexpression network analysis (WGCNA), a systematic biological method, and univariate survival analysis to construct an IRG prognostic index (IRGPI). We then identified the correlation between immune infiltration and the IRGPI. Finally, we examined the possibility of the IRGPI to predict immunotherapy response. The results showed that the IRGPI was an individualized immune-related prognostic index independent of clinical factors that can determine the prognosis and predict the immunotherapy response of patients with ccRCC. A graphical abstract is shown in Figure 1.

\section{Material And Methods Patients And Data Acquisition}

In this study, all database information were obtained from the open-access database and all methods were carried out in according with relevant guidelines and regulations. The RNA-seq, clinicopathological and gene mutation data of $611 \mathrm{ccRCC}$ samples, including 539 cancer tissues and 72 normal tissues, were retrieved from TCGA database (Furthermore, the list of IRGs was obtained from the ImmPort database 0 and InnateDB ().

\section{Functional and Pathway Enrichment Analyses of Immune- related Differentially Expressed Genes (DEGs)}

Difference analysis was used to identify DEGs between 539 cancer and 72 normal tissues from TCGA by the "limma" package of $R$ and Wilcox test, visualized by the "pheatmap" package of $R$. The cutoff values were false discovery rate $(F D R)<0.05$ and $\mid \log _{2}[$ fold change $(F C)] \mid>1$. Overlapping genes between the DEGs and IRGs were selected for further functional enrichment analysis, including Gene Ontology (GO) and Kyoto Encyclopedia of Genes and Genomes (KEGG) analysis. Both analyses were performed by the "clusterProfiler" package of R. Color gradients depict high (red) or low (blue) expression levels. $\mathrm{P}<0.05$ was considered statistically significant.

\section{Identification of Immune-related Hub Genes}

WGCNA was used to describe the associations of gene clusters inside a comprehensive network and evaluate the correlations between gene modules and phenotypes ${ }^{8}$. We evaluated the immune-related 
DEGs and used the R package "WGCNA" to construct different gene coexpression modules. First, the similarity matrix was constructed by Pearson analysis of the immune-related DEGs. Next, an adjacency matrix and a topological overlap matrix (TOM) were obtained according to the power value $(\beta=3)$. A dissimilarity measure (1-TOM) was used to cluster the immune-related DEGs, and a dynamic tree cut algorithm was established for module recognition. For each module, we correlated the module eigengenes (MEs) with clinical features (tumor or normal status). Ultimately, we identified 7 modules by setting the merging soft-thresholding power at 0.25 . We selected 50 immune-related hub genes with a threshold degree (the number of connections between one point and other points in the network) of $>20$.

\section{Identification of Prognosis-related IRGs (PRIRGs)}

We excluded samples from patients with ccRCC with incomplete clinical prognostic information and retained 513 samples with a follow-up time $>30$ days as the entire group. The "caret" package of $R$ was used to randomly divide the $513 \mathrm{ccRCC}$ samples into a training cohort and a testing cohort at a ratio of 2:1. Detailed information is presented in Supplementary Table S1. The training TCGA survival information was used to determine the prognosis-related genes through univariate Cox regression analysis by using "survival" package and "survminer" package of R software, and among 50 immune-related hub genes, 34 significant PRIRGs were selected for further analysis $(P<0.05$, log-rank test). To determine the potential regulatory mechanisms, functional enrichment analysis of the 34 PRIRGs was performed with $R$ software. The gene network of PRIRGs was generated with online tool GeneMANIA () and protein networks were generated with online tool STRING ().

\section{Construction and Validation of the IRGPI}

Through multivariate Cox regression analysis, the genes significantly affecting overall survival (OS) were identified and used to construct an IRGPI based on the PRIRGs. The IRGPI of each sample was calculated as follows: IRGPI = Expression $_{\mathrm{GENE1}}$ * Coefficient $_{\mathrm{GENE} 1}+$ Expression $_{\mathrm{GENE2}}$ * Coefficient $_{\mathrm{GENE2}}+\ldots$

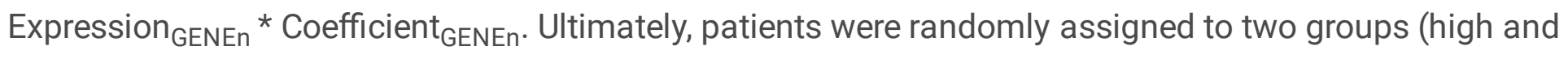
low IRGPI) according to the median IRGPI score in the training group by using "survival" package and "survminer" package of R software. The prognostic power of the IRGPI was evaluated by log-rank tests for survival analysis and the 1-,3-, and 5-year receiver operating characteristic (ROC) curve analyses among the TCGA training $(n=342)$, testing $(n=171)$ and entire cohorts $(n=513)$ by using "timeROC" package of R software. Whether the IRGPI score and clinical factors displayed independent prognostic value for OS was evaluated by univariate and multivariate Cox regression analyses. Moreover, chi-square tests were used to assess the association of the signature with other clinical factors (age, sex, grade and stage) and visualized using the "ComplexHeatmap" package of R software, and nomogram models were drawn. 


\section{Comprehensive Analysis of Immune Characteristics and Immunotherapy Response in Different IRGPI Subgroups}

In the signaling pathway analysis, gene set enrichment analysis (GSEA) based on GO and KEGG analyses was performed to compare the differential gene sets between two IRGPI subgroups. The normalized enrichment score (NES), FDR and adjust P-value were used to adjudicate each enriched pathway. The GO gene sets (c5.go.v7.4.symbols.gmt) and KEGG gene sets (c2.cp.kegg.v7.4.symbols.gmt) in the GSEA database were obtained from .

Cell type identification by estimating relative subsets of RNA transcripts (CIBERSORT) was utilized and iterated 1000 times to quantify the relative proportions of 22 human immune cell subsets in $513 \mathrm{ccRCC}$ samples by using "e1071" package and "preprocessCore" package of R software, and the correlation between the 22 immune cells and prognosis was analyzed. To further define the immune characteristics of the IRGPI subgroups, we quantified the enrichment of 30 immune cell subgroups and immune function in every ccRCC sample based on specific gene marks by the singlesample GSEA (sSGSEA) score and compared the score between two IRGPI subgroups by using "GSVA" package and "GSEABase" package of R software ${ }^{9-11}$.The R packages "ggpubr" then used to visualize the different expression in two IRGPI subgroups.

The expression of some critical immune checkpoints and immunosuppressive genes in different IRGPI subgroups and the correlation between the genes and the IRGPI score were investigated. Tumor mutational burden (TMB) can be utilized to assess the therapeutic effect of immunotherapy in some cancers $^{12}$. We performed correlation analysis between the IRGPI score and TMB by using gene mutation information obtained from TCGA. In addition, we employed the tumor immune dysfunction and exclusion (TIDE) algorithm and calculated microsatellite instability (MSI) obtained from TIDE website (http://tide.dfci.harvard.edu/) to predict the immune response and determine the prognostic value of the IRGPI in patients treated with immunotherapy.

\section{Statistical Analysis}

Statistical analysis or visualization were performed by R software (version 4.1.1). Continuous normally distributed data were compared by t-tests. Categorical variables were compared by chi-square tests. Spearman's coefficient was used to analyze the correlations between TMB and IRGPI score. Univariate KM survival analysis was performed, and the log-rank test was used to analyze differences between the curves. Multivariate survival analysis was performed with Cox proportional hazards models. $\mathrm{P}$ less than 0.05 was considered statistically significant.

\section{Results}

\section{Identification of Immune-related Hub Genes}


In the differential expression analysis of 539 tumor vs. 72 normal tissues, a total of 9459 DEGs were identified (Figure 2A). By intersecting these genes with the lists of IRGs downloaded from ImmPort and InnateDB, 946 differentially expressed IRGs (Table S2) were obtained, of which 780 IRGs were upregulated (red) and 166 were downregulated (blue) in renal cancer tissues compared with normal tissues (Figure 2B, C). The enrichment analysis showed that the 946 differentially expressed IRGs were significantly associated with 2015 GO function terms and 86 KEGG pathways, and the top 30 GO function terms and top 8 KEGG pathways enrichment were visualized in Figure 2D, E. To determine the immune-related hub genes, we visualized the distribution of expression of the differentially expressed IRGs ( $n=946)$ and obtained 7 different modules based on a power of $\beta=3$ and used the dynamic hybrid tree cutting method to merge the highly familiar modules with a minimum module size of 25 and a cutting line $<0.25$ (Figure 3A, B, C). Only one module (brown) was negatively correlated with ccRCC, whereas the green, red, black, blue and yellow modules were positively correlated with ccRCC. The modules contained 137 genes (brown), 61 genes (green), 36 genes (red), 33 genes (black), 534 genes (blue), and 130 genes (yellow) (Figure 3D). Among the above modules significantly related to tumor and normal samples, we obtained 50 immune-related hub genes with a threshold weight $>0.2$ and a threshold degree of $>20$. A total of 34 PRIRGs were correlated with ccRCC patient OS, as determined by univariate Cox regression analysis in the training cohort. ( $P<0.05$, log-rank test) (Figure 3E); therefore, these PRIRGs were further analyzed. GO analysis of the genes showed that they were significantly enriched in the terms $\mathrm{T}$ cell activation (BP), external side of plasma membrane (CC), and cytokine receptor binding (MF) (Figure 4A). KEGG analysis found that the PRIRGs were significantly enriched in cytokine-cytokine receptor interactions and immune responses (Figure 4B). The gene-gene and proteinprotein interaction networks are shown in Figure 4C, D.

\section{IRGPI served as an Independent Prognostic Indicator}

In the training cohort $(n=342)$, multivariate Cox regression analysis of OS was performed for the 34 PRIRGs, and 11 genes (IFNG, XCL1, APOBEC3G, CD86, CXCR3, IL10RA, IL2RG, CD244, SH2D1A, CD3D and FCER1G) significantly affected the OS of ccRCC patients. Then, we constructed a prognostic index calculated by the formula IRGPI= Expression ${ }_{\mathrm{IFNG}}$ * $(0.884)+$ Expression $_{\mathrm{xCL} 1}$ * $(0.307)+$ Expression APOBEC3G $^{*}(0.160)+$ Expression $_{\mathrm{CD} 86} *(-0.263)+$ Expression $_{\mathrm{CXCR3}}$ * $(-0.263)+$ Expression $_{\text {IL10RA }}$ * $(0.156)$ + Expression $_{\mathrm{IL} 2 \mathrm{RG}}$ * $(0.183)+$ Expression $_{\mathrm{CD} 244}$ * $(-0.938)+$ Expression $_{\mathrm{SH} 2 \mathrm{D} 1 \mathrm{~A}}$ * $(-0.376)+$ Expression $_{\mathrm{CD} 3 \mathrm{D}}$ * $(-0.161)+$ Expression $_{\text {FCER1G }}$ * (0.059). Patients were classified based on the median IRGPI score, and the OS of IRGPI ${ }^{\text {high }}$ patients was worse than that of IRGPI low patients in TCGA training $(P<0.001$, log-rank test), testing $(P=0.029$, log-rank test) and entire cohorts $(P<0.001$, log-rank test) (Figure $5 A)$.

Subsequently, the survival prediction reliability of the IRGPI was analyzed by time-dependent ROC curve analysis (Figure 5B). The area under the ROC curve was $0.737,0.719$ and 0.760 for predicting $1-, 3-$, and 5year OS, respectively, in the training cohort, which indicated a moderate accuracy of the IRGPI in predicting survival. 
To further explore the characteristics of the IRGPI score in a large cohort and draw more convincing conclusions, we conducted further experiments in the entire TCGA cohort. Univariate Cox regression showed that age, grade, stage and the IRGPI score were associated with the prognosis of ccRCC. After adjusting for age, sex, grade and stage, multivariate Cox regression analysis confirmed that the IRGPI (HR: 1.566, 95\% Cl: 1.284-1.910, P < 0.001) was an independent prognostic factor (Figure 5C). Chisquare tests were employed to evaluate the association of the IRGPI subgroups and other clinicopathological factors. IRGPI ${ }^{\text {high }}$ was more commonly detected in patients with poor tumor differentiation (grade 3-4), advanced pathological stage (stage $\mathbb{\bigotimes}-\mathbb{Z}$ ), large tumor size and deep local infiltration area(T3-4), distant metastasis(M1) and higher $N$ stage $(P<0.05$, Figure $6 A)$. These findings indicated that the risk signature was well correlated with a variety of clinical factors. The higher the IRGPI score, the poorer the clinical pathological status. Furthermore, a nomogram including clinical factors and IRGPI score for the entire TCGA cohort was generated (Figure 6B, C, D).

\section{Enrichment Analysis}

GSEA identified 18 significant KEGG pathways and $352 \mathrm{GO}$ terms enriched in the IRGPIlow samples, while 21 significant KEGG pathways and $430 \mathrm{GO}$ terms were enriched in the IRGPI ${ }^{\text {high }}$ samples. The top 3 pathways in the high- and low-IRGPI subgroups are shown in Figure 7.

\section{Immune Characteristics of Different IRGPI Subgroups}

To estimate immune cell infiltration in the entire ccRCC cohort, CIBERSORT was adopted to evaluate the relative proportions of 22 tumor-infiltrating immune cell subtypes in different IRGPI subgroups. As shown in Figure 8A, B, the infiltrating immune cells with high proportions in ccRCC included resting memory CD4 T cells, CD 8 T cells and macrophages. Among the 22 immune-infiltrating cells, monocytes and resting mast cells had higher infiltrations in the IRGPI low subgroup, while activated memory CD4 T cells, follicular helper $\mathrm{T}$ (Tfh) cells, regulatory $\mathrm{T}$ cells (Tregs) and $\mathrm{MO}$ macrophages had higher infiltrations in the IRGP ${ }^{\text {high }}$ subgroup $(\mathrm{P}<0.05)$. We analyzed 30 immune-related genomes representing different immune cell types, functions, and pathways (Figure $8 \mathrm{C}$ ). Enrichment of immune signature scores indicated that several immune cell types, including multiple types of dendritic cells (DCs), CD8 T cells, macrophages, T helper (Th) cells, Tfh cells, Th1 cells, Th2 cells, TILs, Tregs and tumor-associated macrophages (TAMs), and several immune terms, including APC costimulation and coinhibition, chemokine receptors (CCRs), checkpoints, cytolytic activity, HLA, inflammation promotion, MHC class I, T cell costimulation and

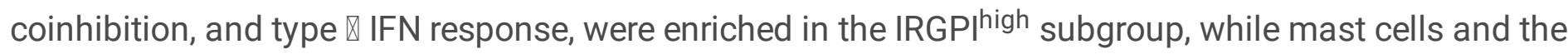
type II IFN response were enriched in the IRGPI ${ }^{\text {low }}$ subgroup. Immunosuppressive cells were present at higher proportions in the IRGPI ${ }^{\text {high }}$ subgroup. In addition, we analyzed the association between the OS of ccRCC patients and 22 immune cell populations: a high abundance of infiltrating resting memory CD $4 T$ cells, neutrophils, monocytes, resting mast cells, M2 macrophages, resting DCs and naïve B cells was related to better survival rates, while a high abundance of infiltrating Tregs, Tfh cells, activated 
memory CD4 T cells, plasma cells, M1 macrophages and M0 macrophages was related to poorer survival rates (Figure S1).

\section{The IRGPI Predicts Response to Immunotherapy}

The expression of immunosuppressive genes was investigated. The expression of PD-1 (PDCD1), PDL2 (PDCD1LG2), CTLA-4, LAG-3, GZMB, BTLA, TIGIT, and TGFB1 was higher in the IRGPI ${ }^{\text {high }}$ subgroup than in the IRGPI ${ }^{\text {low }}$ subgroup and had a positive correlation with the IRGPI score (Figure 9A and Table 1). The above results indicate that the IRGPI ${ }^{\text {low }}$ group was more likely to respond to immunotherapy. TIDE can be used to predict $\mathrm{ICI}$ response and $\mathrm{ICl}$ therapy outcome and to reveal mechanisms of tumor immune escape ${ }^{13,14}$. The correlation among the IRGPI, TIDE score and MSI in cCRCC patients was explored. In our results, the IRGPI ${ }^{\text {high }}$ subgroup had a higher TIDE score than the IRGPIlow subgroup, implying that $\left.I_{R G P}\right|^{\text {high }}$ patients had a higher potential for immune escape and thus were less likely to benefit from immunotherapy than IRGPI ${ }^{\text {low }}$ patients. Moreover, higher tumor TIDE scores are associated with poor patient survival under anti-PD1 or anti-CTLA4 treatments. Additionally, we found that the IRGPI low subgroup had a higher MSI score and a lower T cell dysfunction score, but no statistically significant difference in T cell exclusion between the high and low groups was observed (Figure 9B). TMB is a wellknown immunotherapy biomarker. The results showed that the IRGPI was positively correlated with TMB $(R=0.16, P=0.0048$. Figure $9 C)$, and the $V H L$ and PBRM1 genes had the highest TMB.

Table 1

Correlation between immunosuppressive genes and the IRGPI.

\begin{tabular}{|llllll|}
\hline Gene & rvalue & P value & Gene & rvalue & P value \\
CD274 (PD-L1) & 0.086 & 0.05 & PDCD1 (PD-1) & 0.25 & $\mathbf{6 . 9 E - 0 9}$ \\
\hline TGFB1 & 0.18 & $\mathbf{2 . 7 E - 0 5}$ & TIGIT & 0.28 & $\mathbf{5 . 8 E - 1 1}$ \\
GZMB & 0.17 & $\mathbf{0 . 0 0 0 1 2}$ & LAG3 & 0.34 & $\mathbf{2 . 5 E}-15$ \\
CTLA4 & 0.29 & $\mathbf{3 . 7 E - 1 1}$ & BTLA & 0.20 & $\mathbf{6 . 6 E - 0 6}$ \\
\hline PDCD1LG2 (PD-L2) & $\mathbf{0 . 1 7}$ & $\mathbf{0 . 0 0 0 1 3}$ & HAVCR2 (TIM3) & 0.05 & 0.25 \\
\hline
\end{tabular}

\section{Discussion}

Many ccRCC patients have a poor prognosis because they are initially diagnosed at advanced disease stages despite significant improvements in diagnosis and treatment in recent years ${ }^{15}$. ccRCC is a kind of tumor with high immune infiltration ${ }^{16}$. Immunotherapy has recently emerged as a choice for ccRCC patients, particularly those with metastatic diseases. Accumulating studies have demonstrated that the 
TIME can influence the progression of $\operatorname{ccRCC}^{17,18}$. An increasing number of immune genes have been discovered; however, the immune molecular mechanisms of ccRCC are poorly defined. An accurate prediction of prognosis based on immune genes is essential for guiding patients, individualized monitoring and selecting patients for clinical trials.

In the present study, based on ccRCC immune gene datasets, we identified differentially expressed IRGs. GO function and KEGG pathway analyses were performed to explore the role of these differentially expressed IRGs in ccRCC. As expected, the results revealed that genes were enriched in processes such as the adaptive immune response, immunoglobulin complex, antigen binding and cytokine-cytokine receptor interaction. Based on the results of the univariate Cox analysis, we used WGCNA to identify 34 PRIRGs affecting patient OS. GO function and KEGG pathway analyses revealed that these genes were enriched in $T$ cell activation and related to the terms external side of plasma membrane, cytokine receptor binding and cytokine-cytokine receptor interaction. Previous studies have shown that chemokines and their receptors can recruit immune cell subsets into the tumor microenvironment ${ }^{19}$. Chemokines also influence cancer progression and therapy in ccRCC ${ }^{20}$. This evidence suggests that the 34 immune-related hub genes can function not only as potential biomarkers for prognosis but also as therapy targets for ccRCC.

We randomly divided the $513 \mathrm{ccRCC}$ patients into training and testing cohorts for validation of the IRGPI model we constructed based on 11 survival-associated differentially expressed IRGs (IFNG, XCL1, APOBEC3G, CD86, CXCR3, IL10RA, IL2RG, CD244, SH2D1A, CD3D and FCER1G) to draw a more reliable conclusion. The IRGPI proved to be a valid prognostic immune-related biomarker for CCRCC, with worse survival in IRGPI ${ }^{\text {high }}$ patients and better survival in IRGPIlow patients in the three TCGA cohorts. Although IFNG enhanced immune function, it promoted T cell exhaustion through PDL1, and its functions in cancer cells and immune cells were opposite ${ }^{21}$. High expression of IFNG was associated with poor prognosis in CCRCC $^{22}$. XCL1 is a $\mathrm{C}$ class chemokine, that is produced by $\mathrm{T}$ and natural killer cells during inflammatory responses $^{23}$. APOBEC3G was overexpressed in renal cancer ${ }^{24}$. The APOBEC family can drive tumor evolution and may induce recurrence, metastasis, and/or therapy resistance ${ }^{25}$. The interaction between CD86 and ligand is the main factor inducing T lymphocyte proliferation and IL-2 production. CXCR3 is the receptor of CXCL10, and alternative splicing of CXCR3 (producing CXCR3-A and CXCR3-B) is partially related to the different functions (tumor-suppressive and tumor-promoting functions) of CXCL10 in renal cancer. Downregulation of CXCR3-B promoted renal cancer cells proliferation and migration ${ }^{26}$. Interleukin 10 receptor subunit alpha (IL10RA) is mainly expressed on most hematopoietic cell and functions as an anti-inflammatory factor ${ }^{27}$. FCER1G was associated with the progression and poor prognosis of ccRCC $^{28}$. CD244, as a receptor of NK cells, plays important roles in the anticancer function of NK cells. In the IRGPI calculation formula, the coefficients of IFNG, XCL1, APOBEC3G, IL10RA, IL2RG and FCER1G were positive, indicating that high expression of the above genes had a worse outcome in cCRCC patients. However, the coefficients of CD86, CXCR3, CD244, SH2D1A and CD3D were negative, suggesting that the above genes may function as protective factors for cCRCC patients. In summary, IRGPI could be used as a biomarker associated with immune cell dysfunction(exhaustion) and tumor cell proliferation and migration. 
The IRGPI presented moderate value in predicting the prognosis of ccRCC patients independent of other clinical factors. Furthermore, a close correlation was found between the IRGPI and some clinical factors. To further improve the correctness of the prognosis model, a nomogram incorporating the IRGPI score and clinical factors was constructed and verified, and this nomogram could also predict the OS of patients to guide clinical practice.

To understand the molecular mechanism of the two subtype groups, GSEA was used to analyze the differences in activated pathways. In the GO and KEGG GSEA, active immune responses and chemokine signaling pathways were found to be enriched in the IRGPI ${ }^{\text {high }}$ subgroup. The JAK/STAT signaling pathway downstream of the chemokine signaling pathway was also enriched in the IRGPI ${ }^{\text {high }}$ subgroup, indicating that inflammation may be involved in tumor progression. The enrichment of immune response activation in the IRGPI $\left.\right|^{\text {high }}$ subgroup seems to contradict the worse prognosis of this group. However, the phenotypic and functional profiles of immune cells vary depending on the tumor subtype and the TIME, and the role of immune cells in $\mathrm{cCRCC}$ and the surrounding immune environment needs to be further studied.

Immune cell infiltration plays an important role in tumor progression and metastasis. Understanding the landscape of the TIME could help to find new ways to treat cCRCC or to alter the TIME to improve the effectiveness of immunotherapy. The immune characteristics of the IRGPI ${ }^{\text {high }}$ subgroup indicated that the inability of TILs to mediate antitumor functions is likely due to the overrepresentation of immunosuppressive cells [e.g., Tfh cells, uncommitted (M0) macrophages, HLA-G/E-expressing cells, Tregs and TAMs] and promotion of inflammation. T cells exposed to persistent antigen and/or inflammatory signals can develop defects and become anergic ${ }^{29}$. In contrast to almost all other tumors, cCRCC tumors with increased infiltration of $C D 8+T$ cells and $C D 4+T$ cells have high tumor grade and poor prognosis ${ }^{30,31}$. Macrophages play an immunosuppressive role in cCRCC patients by increasing the activity of Tregs ${ }^{32}$. It has been found that increased expression of nonclassical HLA class I molecules (HLA-G and HLA-E) negatively affects T cell function ${ }^{33}$. TAMs are the main sources of vascular permeability factors, immunosuppressive mediators and proinflammatory cytokines in the TIME. In cCRCC, the accumulation of $\mathrm{C} 1 \mathrm{q}+$ TAMs were correlated with an exhausted T cell phenotype and poor OS 34 . These findings support our study. The immune escape of tumors is an essential step in the process of cancer progression to evade the immune system and prevent the body from producing an effective antitumor response. In addition to immunosuppressive cells, immunosuppressive molecules are also involved. In this study, the expression of most immunosuppressive genes was higher in the IRGPI ${ }^{\text {high }}$ subgroup and had a positive correlation with the IRGPI score. The expression of immunosuppressive molecules was also a characteristic of T cell dysfunction. Increased expression of PD-1 was found to be associated with high-risk RCC tumors, and immune cell PD-1 may promote cancer progression by contributing to immune dysfunction ${ }^{35}$. Since the expression of TGFB1 and HAVCR2 was much higher than that of PD-1 in the TIME, TGFB1 and HAVCR2 may be better targets for immunotherapy in ccRCC. 
Since the composition of immune cell subtypes and the expression of immunosuppressive molecules were different between the two IRGPI subgroups, IRGPI might reflect different immune benefits from ICI therapy (anti-PD1 and anti-CTLA4 therapy) identified with TIDE. In our study, TIDE prediction showed that IRGPI ${ }^{\text {high }}$ patients had a lower ICl response, possibly because of immune evasion via T cell dysfunction. $\mathrm{MSI}$ is a significant factor affecting $\mathrm{ICI}$ therapy. There is evidence indicating that MSI is positively correlated with survival, and patients with high MSI were predicted to benefit from novel immunotherapies in clinical trials ${ }^{36,37}$. Studies have revealed that TMB is a biomarker for predicting immunotherapy response in some cancer types ${ }^{12,38}$. A recent study discovered that high TMB is correlated with poor prognosis and might inhibit immune infiltration in $\operatorname{cCRCC}^{39}$. Based on the above evidence, we speculate that our prediction model based on PRIRGs may serve as a reliable immunerelated tool for cancer ICl therapy.

In conclusion, the IRGPI is a prognostic index independent of clinical factors for estimating the prognosis in ccRCC patients and might predict immunotherapy response in ccRCC patients. The composite prognostic nomogram we established integrating an IRG signature with clinical factors may provide a promising tool for clinical practice.

\section{Declarations}

\section{Author Contributions.}

Fengping $\mathrm{Ji}$ and Xin Liu conceived and designed the study. Fengping Ji, Yanping Zhang and Erpeng Liu analyzed the data. Fengping Ji wrote the draft of the manuscript. Jianguo Wen edited the manuscript. All authors read and approved the final manuscript.

\section{Conflicts of Interest.}

All authors have no conflicts of interest to declare.

\section{References}

1. Gitlitz, B.J. \& Figlin, R.A. Cytokine-based therapy for metastatic renal cell cancer. The Urologic clinics of North America 30, 589-600 (2003).

2. Kotecha, R.R., Motzer, R.J. \& Voss, M.H. Towards individualized therapy for metastatic renal cell carcinoma. Nature reviews. Clinical oncology 16, 621-633 (2019).

3. Motzer, R.J., et al. Nivolumab plus ipilimumab versus sunitinib in first-line treatment for advanced renal cell carcinoma: extended follow-up of efficacy and safety results from a randomised, controlled, phase 3 trial. The Lancet. Oncology 20, 1370-1385 (2019).

4. Taube, J.M., et al. Implications of the tumor immune microenvironment for staging and therapeutics. Modern pathology: an official journal of the United States and Canadian Academy of Pathology, Inc 
31, 214-234 (2018).

5. Xu, W.H., et al. Prognostic value and immune infiltration of novel signatures in clear cell renal cell carcinoma microenvironment. Aging 11, 6999-7020 (2019).

6. Wang, X., et al. An Immune-Related Gene Prognostic Index for Triple-Negative Breast Cancer Integrates Multiple Aspects of Tumor-Immune Microenvironment. Cancers 13(2021).

7. Havel, J.J., Chowell, D. \& Chan, T.A. The evolving landscape of biomarkers for checkpoint inhibitor immunotherapy. Nature reviews. Cancer 19, 133-150 (2019).

8. Langfelder, P. \& Horvath, S. WGCNA: an R package for weighted correlation network analysis. BMC bioinformatics 9, 559 (2008).

9. He, Y., Jiang, Z., Chen, C. \& Wang, X. Classification of triple-negative breast cancers based on Immunogenomic profiling. Journal of experimental \& clinical cancer research: CR 37, 327 (2018).

10. Hänzelmann, S., Castelo, R. \& Guinney, J. GSVA: gene set variation analysis for microarray and RNAseq data. BMC bioinformatics 14, 7 (2013).

11. Dai, T., et al. Regulation Network and Prognostic Significance of Aldo-Keto Reductase (AKR) Superfamily Genes in Hepatocellular Carcinoma. Journal of hepatocellular carcinoma 8, 997-1021 (2021).

12. Liu, L., et al. Combination of TMB and CNA Stratifies Prognostic and Predictive Responses to Immunotherapy Across Metastatic Cancer. Clin Cancer Res 25, 7413-7423 (2019).

13. Jiang, P., et al. Signatures of T cell dysfunction and exclusion predict cancer immunotherapy response. Nature medicine 24, 1550-1558 (2018).

14. Chen, Y., Li, Z.Y., Zhou, G.Q. \& Sun, Y. An Immune-Related Gene Prognostic Index for Head and Neck Squamous Cell Carcinoma. Clin Cancer Res 27, 330-341 (2021).

15. Choueiri, T.K. \& Motzer, R.J. Systemic Therapy for Metastatic Renal-Cell Carcinoma. The New England journal of medicine 376, 354-366 (2017).

16. Yoshihara, K., et al. Inferring tumour purity and stromal and immune cell admixture from expression data. Nature communications 4, 2612 (2013).

17. Singh, D. Current updates and future perspectives on the management of renal cell carcinoma. Life sciences $264,118632(2021)$.

18. Gu, Y.Y., et al. Development and validation of an immune prognostic classifier for clear cell renal cell carcinoma. Cancer biomarkers: section A of Disease markers 27, 265-275 (2020).

19. Nagarsheth, N., Wicha, M.S. \& Zou, W. Chemokines in the cancer microenvironment and their relevance in cancer immunotherapy. Nature reviews. Immunology 17, 559-572 (2017).

20. Wu, Z., et al. Characterization of the Prognostic Values of the CXCR1-7 in Clear Cell Renal Cell Carcinoma (ccRCC) Microenvironment. Frontiers in molecular biosciences 7, 601206 (2020).

21. Benci, J.L., et al. Opposing Functions of Interferon Coordinate Adaptive and Innate Immune Responses to Cancer Immune Checkpoint Blockade. Cell 178, 933-948.e914 (2019). 
22. Lin, J., et al. Identification of biomarkers related to CD8(+) T cell infiltration with gene co-expression network in clear cell renal cell carcinoma. Aging 12, 3694-3712 (2020).

23. Lei, Y. \& Takahama, Y. XCL1 and XCR1 in the immune system. Microbes and infection 14, 262-267 (2012).

24. Komohara, Y., et al. Expression of APOBEC3G in kidney cells. Tissue antigens 69, 95-98 (2007).

25. Olson, M.E., Harris, R.S. \& Harki, D.A. APOBEC Enzymes as Targets for Virus and Cancer Therapy. Cell chemical biology 25, 36-49 (2018).

26. Datta, D., et al. Calcineurin inhibitors modulate CXCR3 splice variant expression and mediate renal cancer progression. Journal of the American Society of Nephrology: JASN 19, 2437-2446 (2008).

27. Shouval, D.S., et al. Interleukin 10 receptor signaling: master regulator of intestinal mucosal homeostasis in mice and humans. Advances in immunology 122, 177-210 (2014).

28. Chen, L., et al. Co-expression network analysis identified FCER1G in association with progression and prognosis in human clear cell renal cell carcinoma. International journal of biological sciences 13 , 1361-1372 (2017).

29. Díaz-Montero, C.M., Rini, B.I. \& Finke, J.H. The immunology of renal cell carcinoma. Nature reviews. Nephrology 16, 721-735 (2020).

30. Fridman, W.H., Zitvogel, L., Sautès-Fridman, C. \& Kroemer, G. The immune contexture in cancer prognosis and treatment. Nature reviews. Clinical oncology 14, 717-734 (2017).

31. Braun, D.A., et al. Beyond conventional immune-checkpoint inhibition - novel immunotherapies for renal cell carcinoma. Nature reviews. Clinical oncology 18, 199-214 (2021).

32. Geissler, K., et al. Immune signature of tumor infiltrating immune cells in renal cancer. Oncoimmunology 4, e985082 (2015).

33. Kochan, G., Escors, D., Breckpot, K. \& Guerrero-Setas, D. Role of non-classical MHC class I molecules in cancer immunosuppression. Oncoimmunology 2, e26491 (2013).

34. Roumenina, L.T., et al. Tumor Cells Hijack Macrophage-Produced Complement C1q to Promote Tumor Growth. Cancer immunology research 7, 1091-1105 (2019).

35. Thompson, R.H., et al. PD-1 is expressed by tumor-infiltrating immune cells and is associated with poor outcome for patients with renal cell carcinoma. Clin Cancer Res 13, 1757-1761 (2007).

36. Hause, R.J., Pritchard, C.C., Shendure, J. \& Salipante, S.J. Classification and characterization of microsatellite instability across 18 cancer types. Nature medicine 22, 1342-1350 (2016).

37. Bonneville, R., et al. Landscape of Microsatellite Instability Across 39 Cancer Types. JCO precision oncology 2017(2017).

38. Yarchoan, M., Hopkins, A. \& Jaffee, E.M. Tumor Mutational Burden and Response Rate to PD-1 Inhibition. The New England journal of medicine 377, 2500-2501 (2017).

39. Zhang, C., Li, Z., Qi, F., Hu, X. \& Luo, J. Exploration of the relationships between tumor mutation burden with immune infiltrates in clear cell renal cell carcinoma. Ann Trans/ Med 7, 648 (2019). 

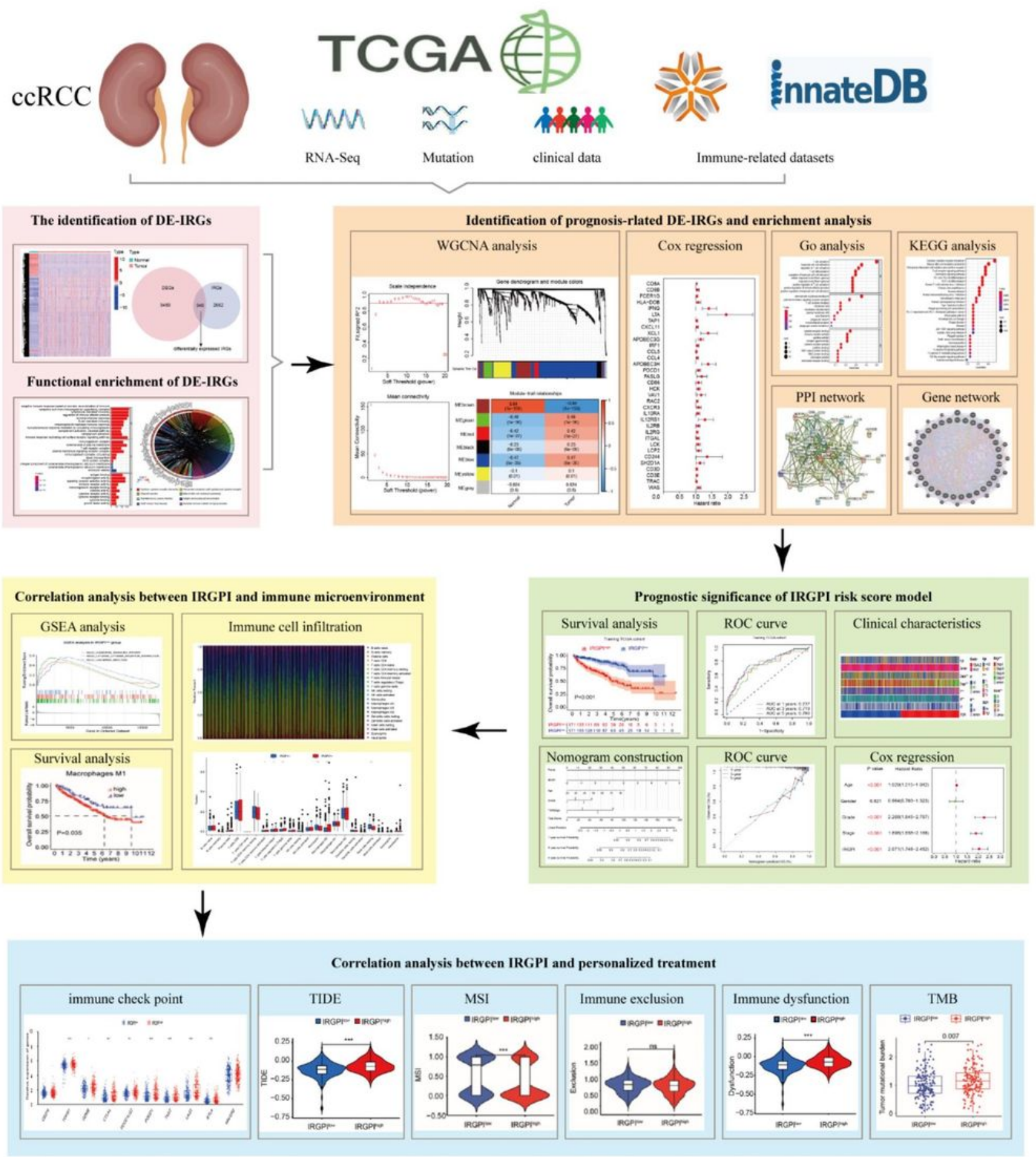

\section{Figure 1}

Graphical abstract 

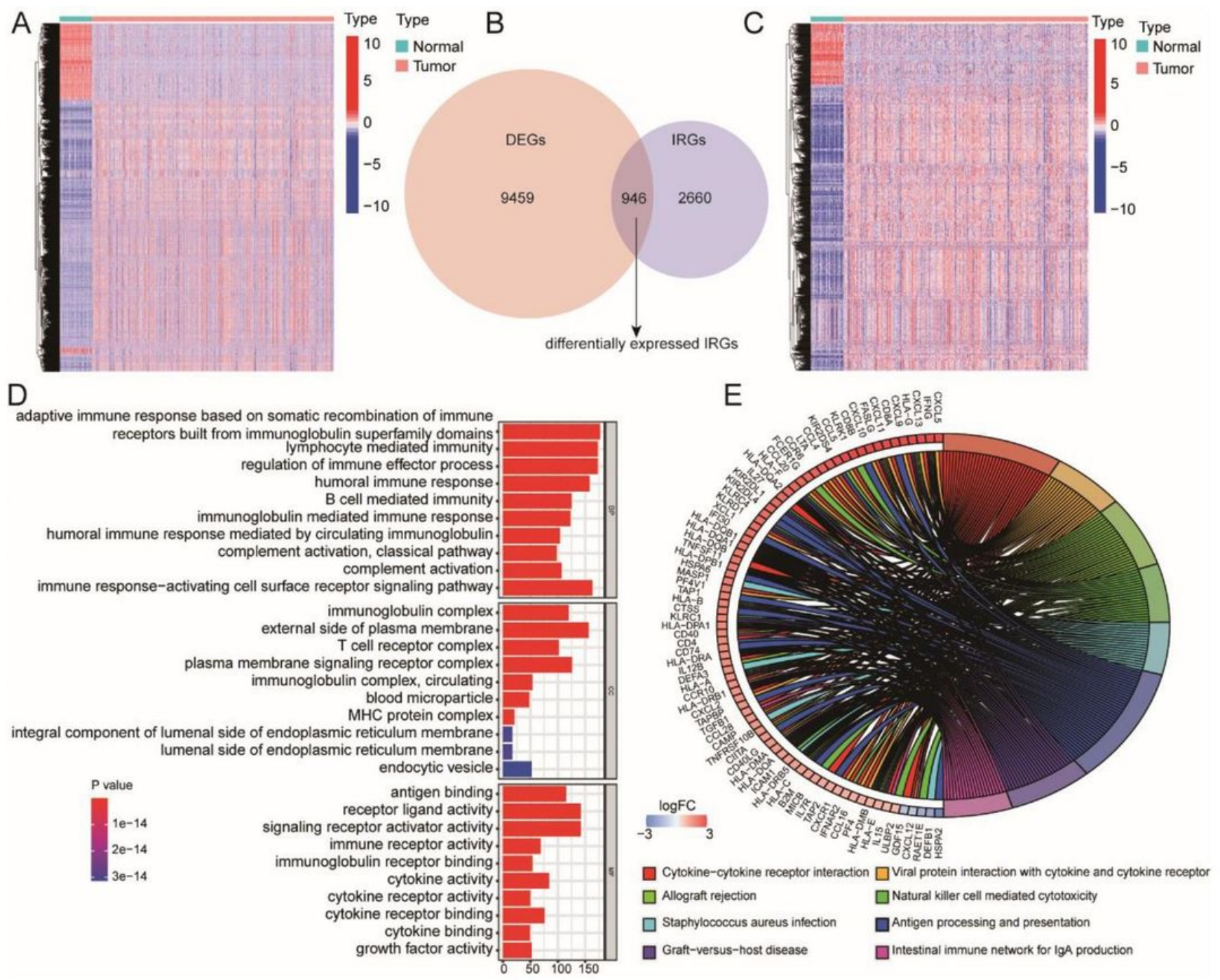

\section{Figure 2}

Enrichment analyses of differentially expressed IRGs. (A) Heat map of 9459 DEGs. (B) Venn diagram visualizing the intersection of the DEGs and IRGs. (C) Heat map of 946 differentially expressed IRGs between ccRCC and normal tissues. (D, E) GO and KEGG enrichment analyses of the 946 differentially expressed IRGs. IRGs, immune-related genes; DEGs, differentially expressed genes; ccRCC, clear cell renal cell carcinoma; GO, Gene Ontology; KEGG, Kyoto Encyclopedia of Genes and Genomes. 
A

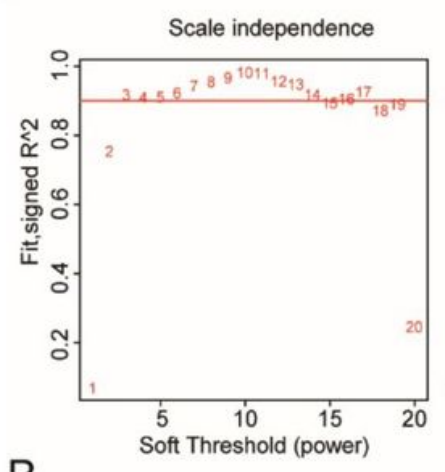

B

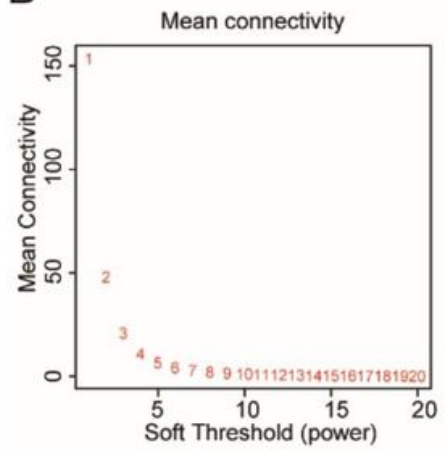

C

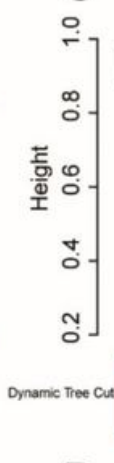

D

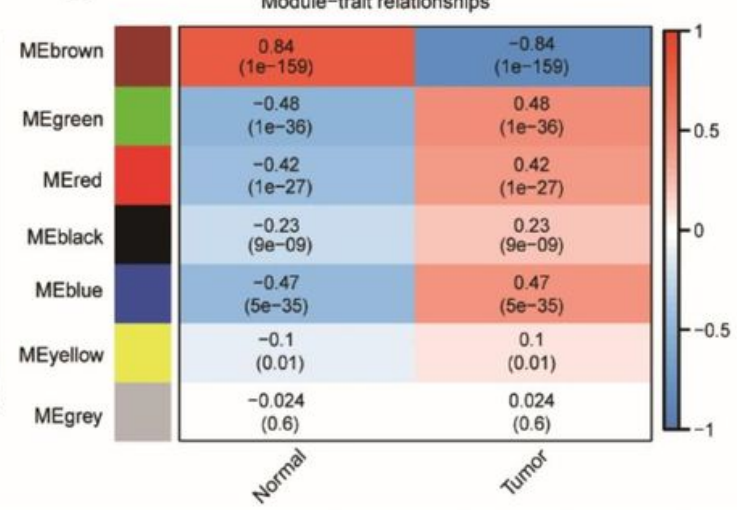

$\mathrm{E}$

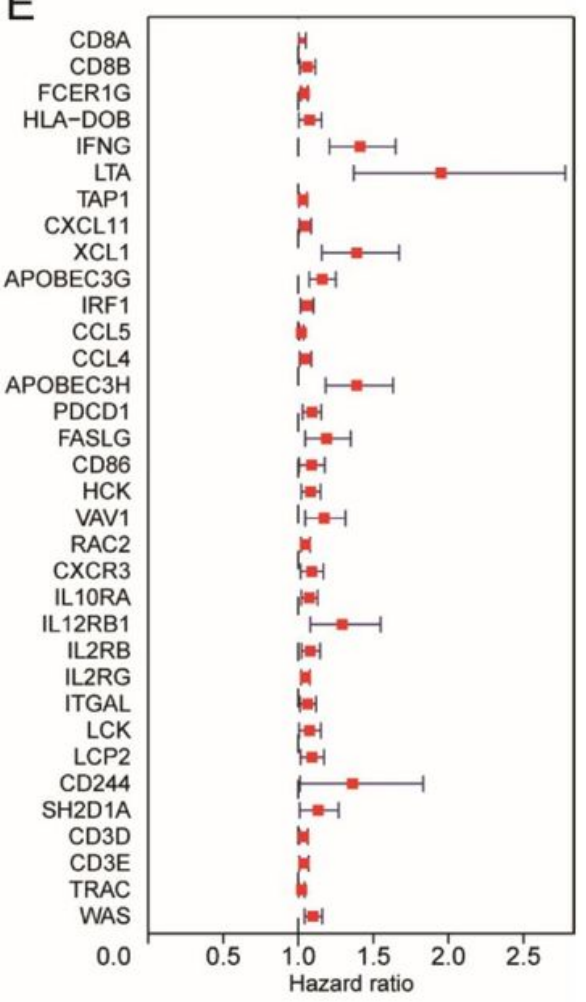

Figure 3

Identification of prognosis-related and immune-related hub genes (PRIRGs). (A, B) Identification of softthresholding powers. (C) The cluster dendrogram of differentially expressed IRGs based on difference metric (1-TOM) clustering. (D) Module-trait (tumor and normal) associations. The correlation coefficient and $P$ values are shown in each cell. (E) Forest plot showing the distribution of prognosis-related hub gene hazard ratios. TOM, topological overlap matrix. 

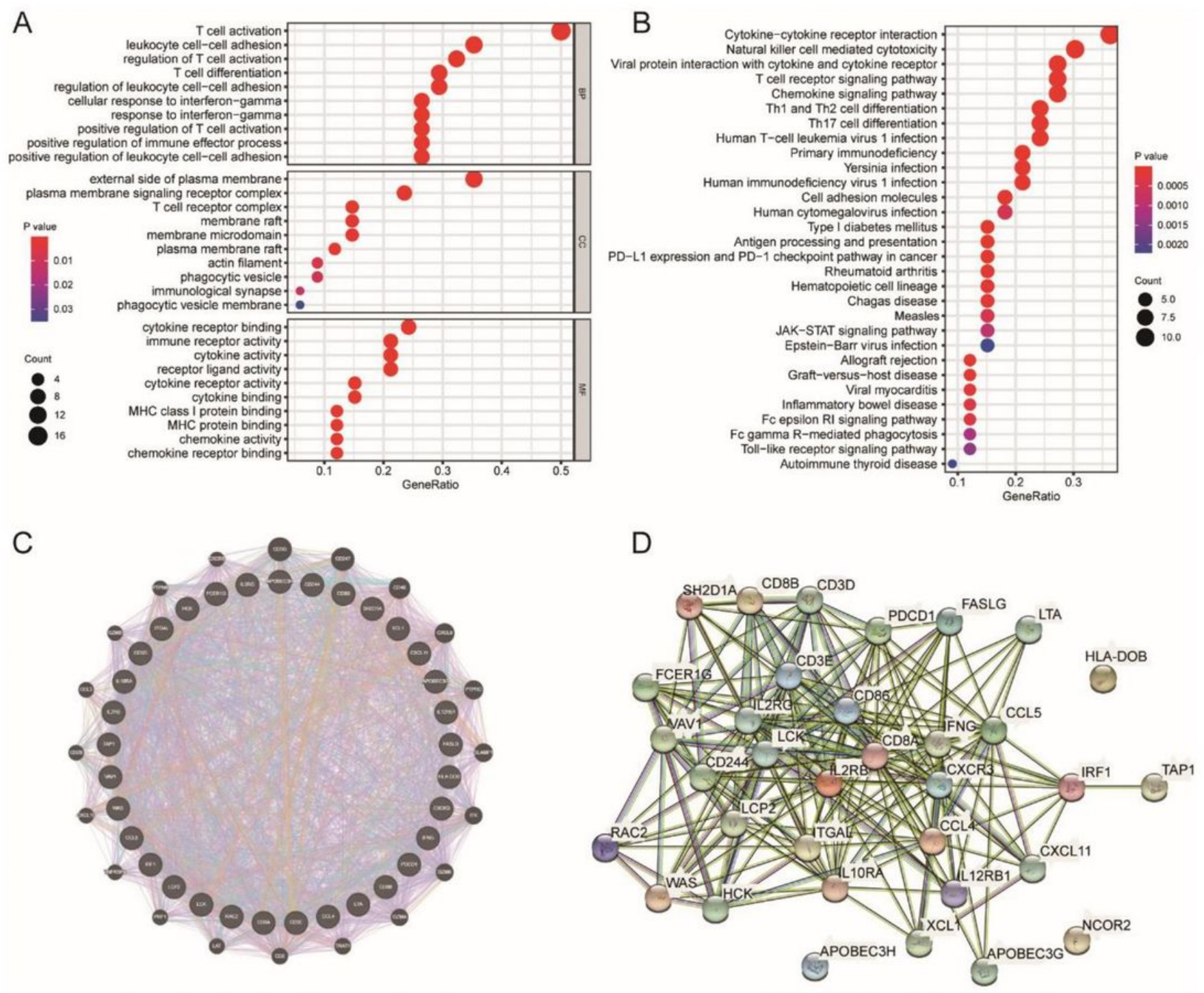

Figure 4

Enrichment analysis of prognosis-related and immune-related hub genes (PRIRGs). (A) GO enrichment analysis of PRIRGs. (B) KEGG enrichment analysis of PRIRGs. (C) The gene network associated with the 34 PRIRGs, generated with GeneMANIA. (D) Network diagram of interactions between proteins encoded by 34 PRIRGs, as generated with STRING. GO, Gene Ontology; KEGG, Kyoto Encyclopedia of Genes and Genomes. 
A

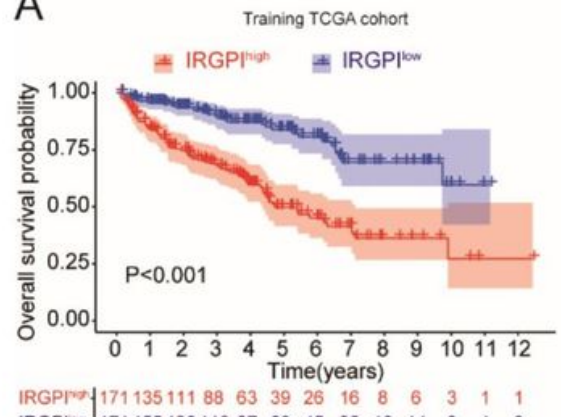

\begin{tabular}{l|l|lllllllll} 
IRGPFo & 17113511188 & 63 & 39 & 26 & 16 & 8 & 6 & 3 & 1 & 1 \\
IRGPIow & 171155128110 & 87 & 63 & 45 & 25 & 18 & 14 & 3 & 1 & 0
\end{tabular}

B

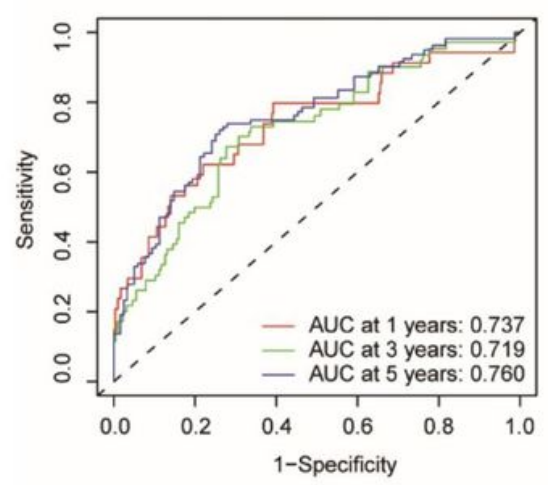

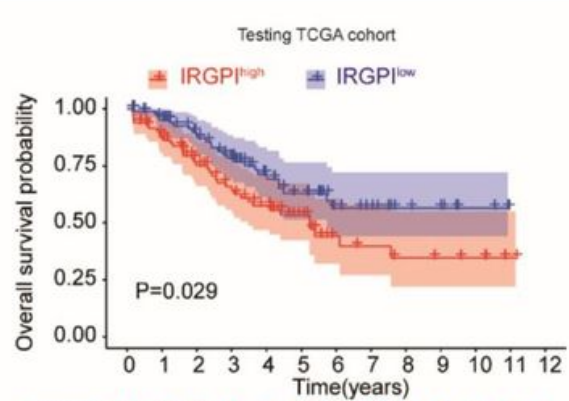

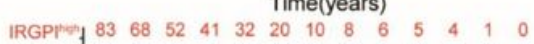
\begin{tabular}{l|lllllllllllll} 
IRGPPOr: & 88 & 77 & 64 & 51 & 35 & 27 & 17 & 13 & 9 & 6 & 3 & 0 & 0 \\
\hline
\end{tabular}

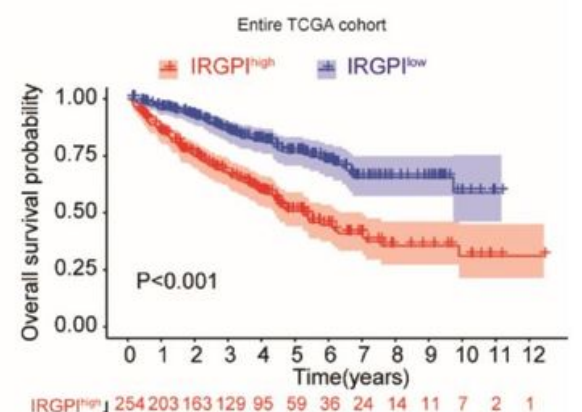

IRGPI:- $25923219216112290 \quad 62 \quad 38 \quad 27 \quad 20 \quad 6 \quad 1 \quad 0$
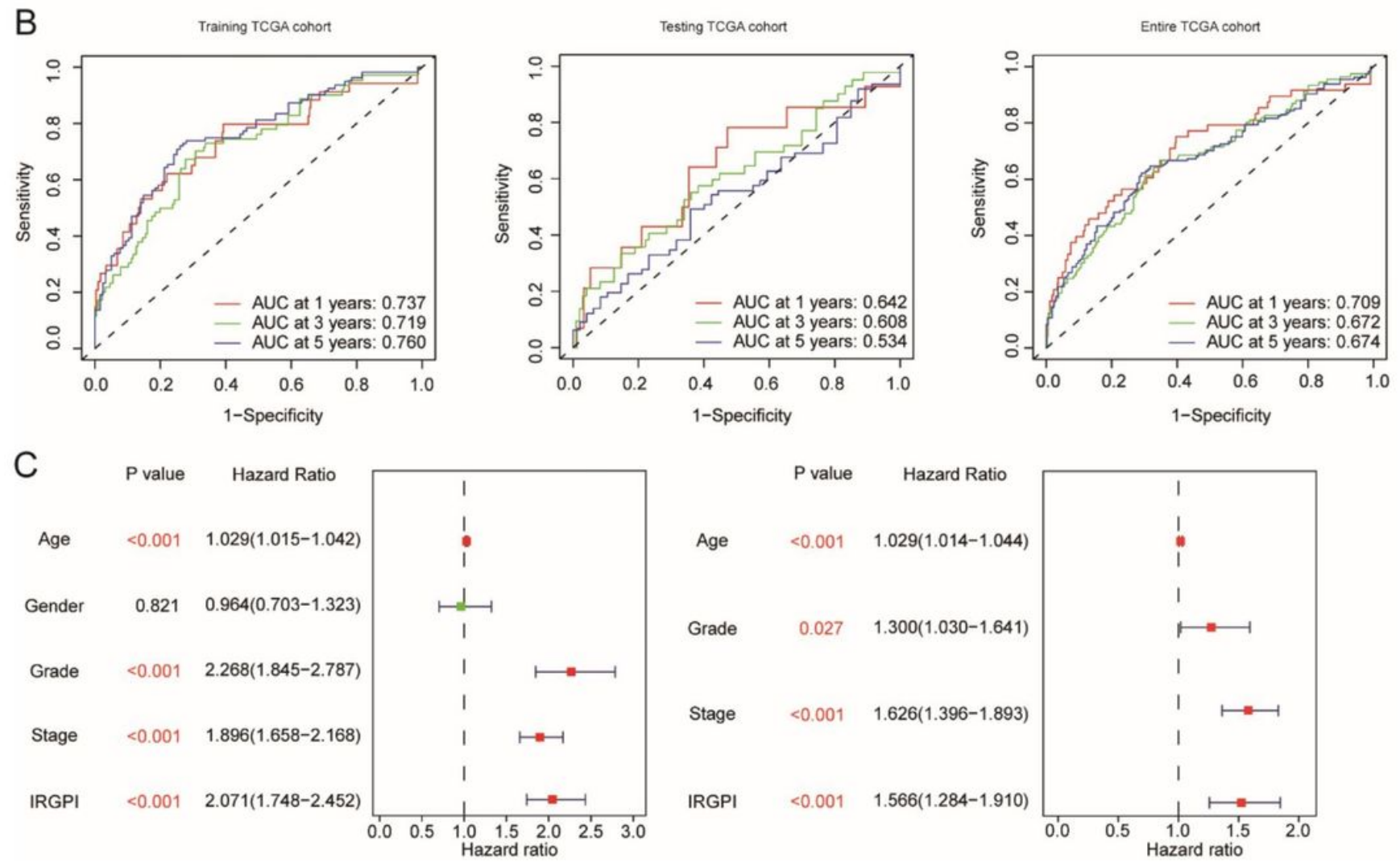

\section{Figure 5}

Prognostic analysis of different IRGPI subgroups. (A) Kaplan-Meier survival analysis of the IRGPI ${ }^{\text {high }}$ and IRGPI low groups in the training, testing and entire TCGA cohorts. (B) ROC curve analysis of the IRGPIhigh and IRGPI ${ }^{\text {low }}$ groups in the training, testing and entire TCGA cohorts. (C) Univariate Cox analysis of clinicopathological factors and the IRGPI score and multivariate Cox analysis of the factors significant in the univariate Cox analysis. IRGPI, immune-related genes prognostic index; ROC, receiver operating characteristic. 


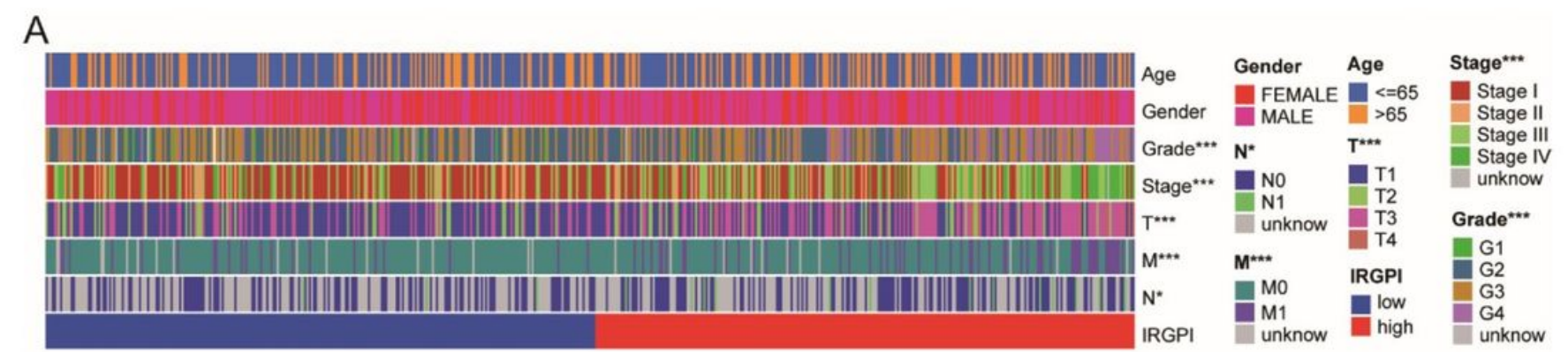

B

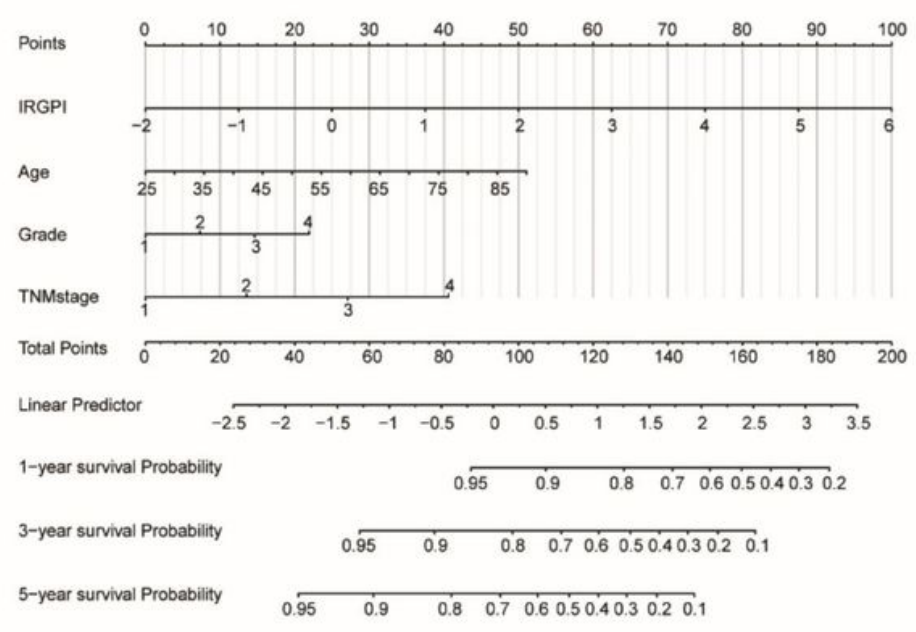

$\mathrm{D}$

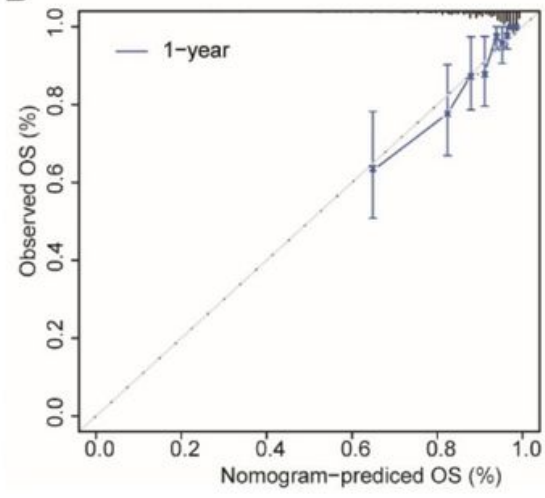

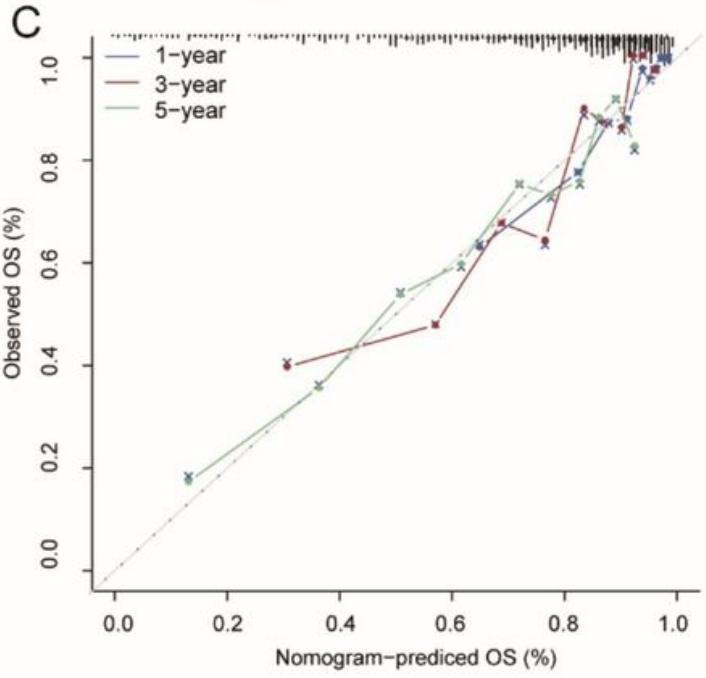
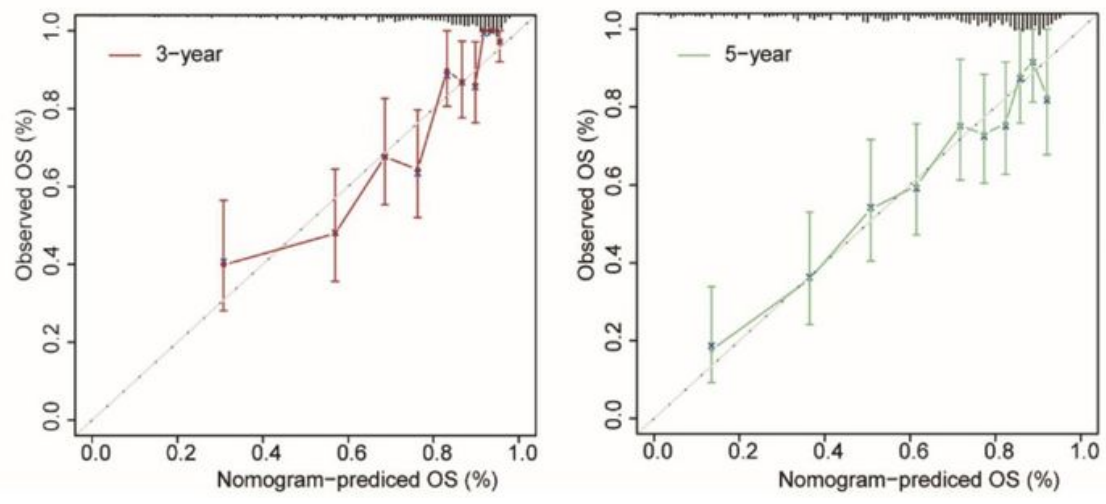

\section{Figure 6}

Correlation of clinical factors with the IRGPI and nomogram plots for the prediction of patient survival. (A) Clinical factor of different IRGPI subgroups. Significant differences between the high and low groups were assessed using the chi-square test $\left({ }^{*} P<0.05,{ }^{*} * \mathrm{P}<0.001\right)$. Age, sex, stage, grade, and $T, N$ and $M$ stages are shown as patient annotations. (B) Nomogram combining the IRGPI score with clinical factors predicts survival rates at 1, 3 and 5 years. (C, D) Calibration chart showing the prediction accuracy of the nomogram. IRGPI, immune-related genes prognostic index. 
A

GSEA analysis in IRGPI $\left.\right|^{\text {inh }}$ group

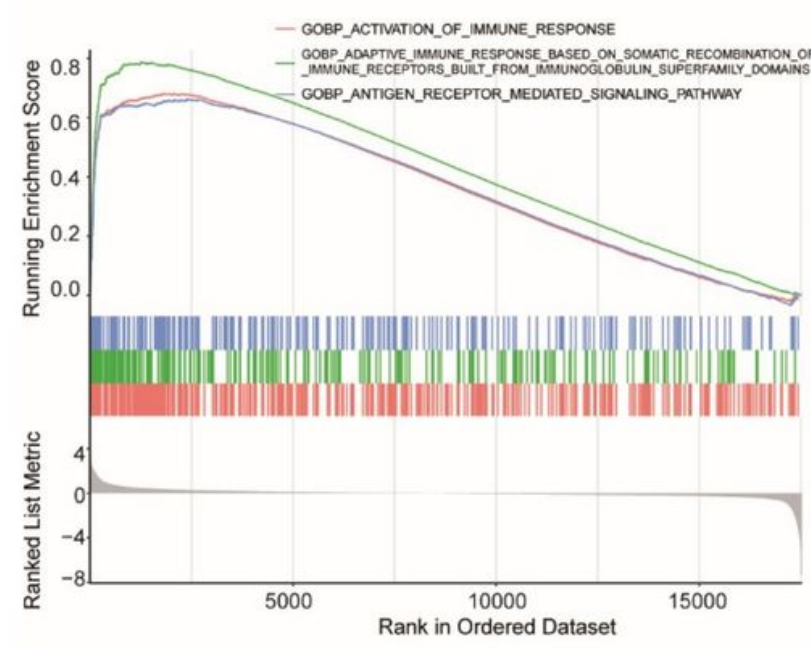

B

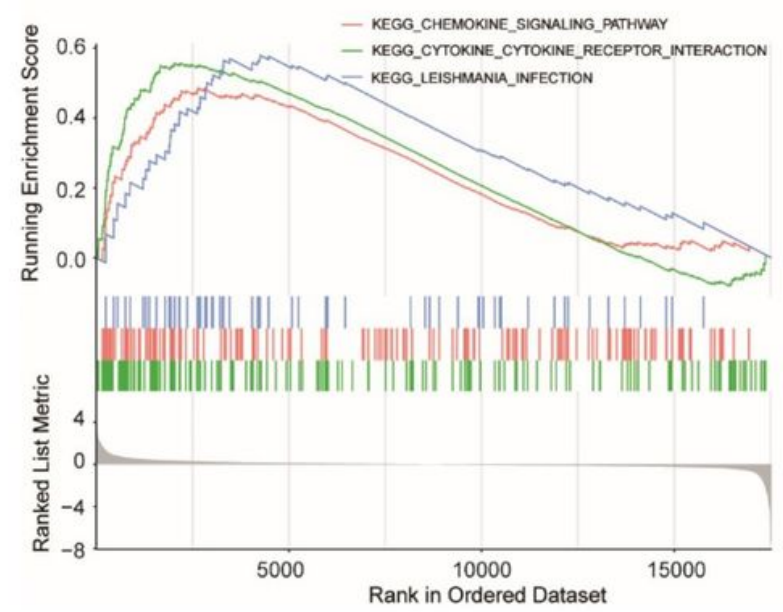

C

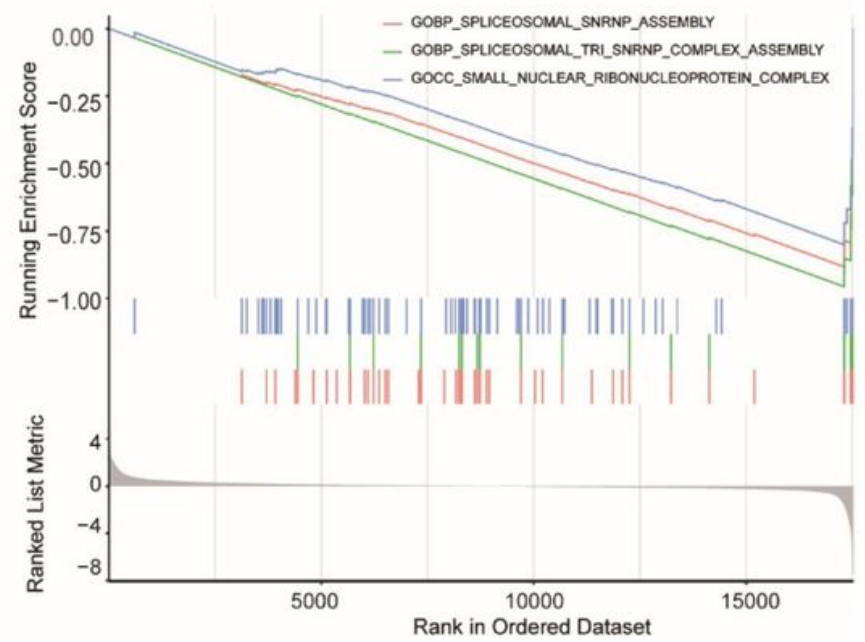

D

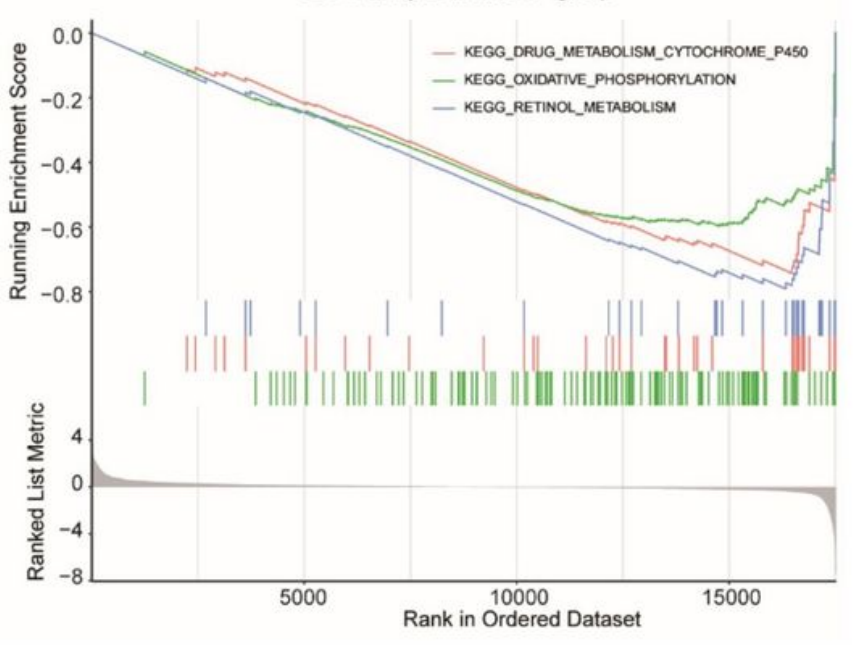

\section{Figure 7}

Gene sets enriched in high and low IRGPI groups. (A, B) Gene sets enriched in the IRGPhigh subgroup. (C, D) Gene sets enriched in the IRGPI ${ }^{\text {low }}$ subgroup. IRGPI, immune-related genes prognostic index. 

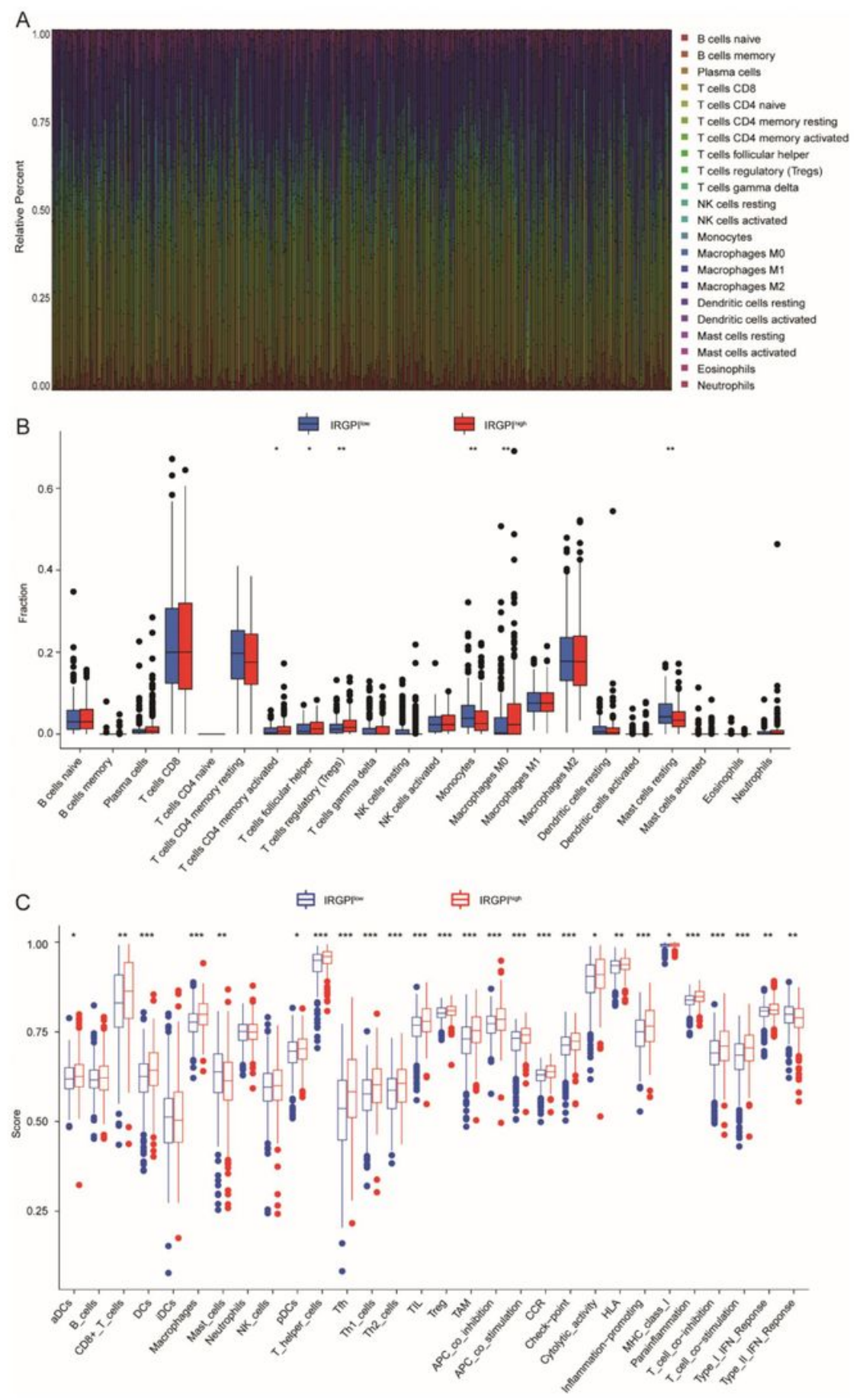

\section{Figure 8}

Immune cell subtypes in different IRGPI groups. (A, B) The proportions of immune cell subtypes in different IRGPI subgroups. The scattered dots represent the immune scores of the two subgroups. The bottom, middle and top of the boxes are the 25th, 50th and 75th percentiles, respectively. ${ }^{*} P<0.05$, $\left.{ }^{\star} \times P<0.01\right)$. (C) Immune signature scores in different IRGPI subgroups $\left({ }^{*} P<0.05,{ }^{*} \times P<0.01,{ }^{\star}{ }^{*} P<0.001\right)$. IRGPI, immune-related genes prognostic index. 

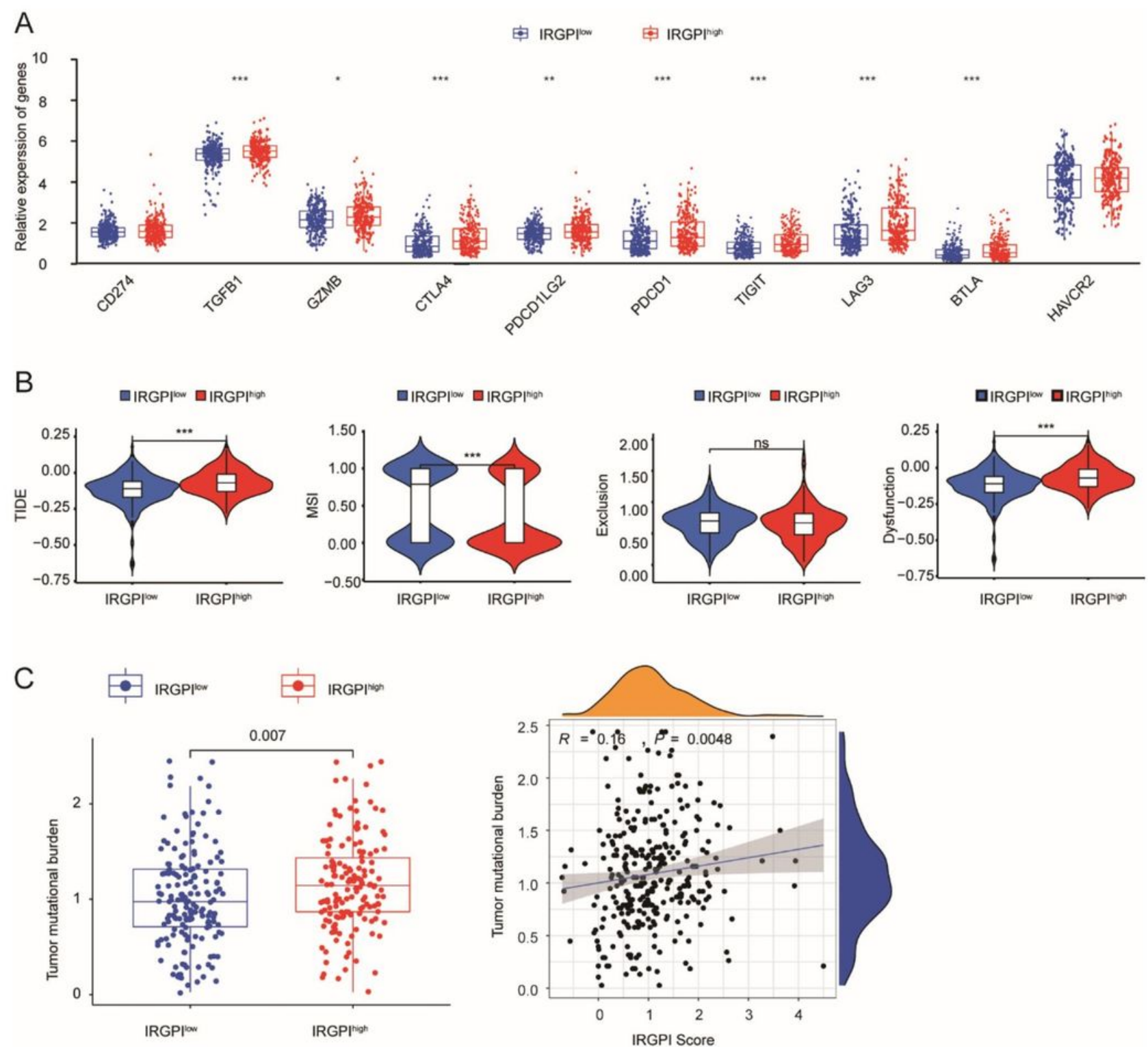

\section{Figure 9}

The prognostic value of the IRGPI in patients receiving immunotherapy. (A) Immunosuppressive genes in different IRGPI subgroups. (B) TIDE score, MSI, and T cell exclusion and dysfunction scores in the high and low IRGPI subgroups (ns: not significant, ${ }^{* \star *} \mathrm{P}<0.001$ ). (C) Correlation between TMB and the IRGPI. IRGPI, immune-related genes prognostic index; TIDE, tumor immune dysfunction and exclusion; $\mathrm{MSI}$, microsatellite instability; TMB, tumor mutational burden.

\section{Supplementary Files}


This is a list of supplementary files associated with this preprint. Click to download.

- supplementaryfigureandtables.pdf 